Supplement of

\title{
Impact of a new emission inventory on CAM5 simulations of aerosols and aerosol radiative effects in eastern China
}

T. Fan et al.

5 Correspondence to: T. Fan (fantianyi@bnu.edu.cn)

\section{Emission}

Table S1. Mapping the MEIC emission to CAM5-MAM3 emission input data in China.

\begin{tabular}{|c|c|c|c|c|c|}
\hline Species & Elevation & $\begin{array}{l}\text { Sectors in } \\
\text { CAM5 }\end{array}$ & $\begin{array}{c}\text { AR5 emission rates }{ }^{\mathrm{e}} \\
(\mathrm{Gg} / \text { year })\end{array}$ & $\begin{array}{l}\text { Sectors in MEIC and } \\
\text { mapping treatment }\end{array}$ & $\begin{array}{c}\text { MEIC emission rates }{ }^{\mathrm{f}} \\
(\mathrm{Gg} / \text { year })\end{array}$ \\
\hline \multirow[t]{10}{*}{$\mathrm{SO}_{2}$} & Surface & dom & 1692.2 & res $\mathrm{SO}_{2}$ x $97.5 \%$ & 1593.3 \\
\hline & & tra & 289.0 & $\operatorname{tra} \mathrm{SO}_{2} \times 97.5 \%$ & 101.1 \\
\hline & & awb & 29.9 & $-{ }^{c}$ & 29.9 \\
\hline & & wst & 0.0 & - & 0.0 \\
\hline & & $\operatorname{shp}$ & 11.2 & - & 11.2 \\
\hline & Elevated & ene & 7946.7 & pow $\mathrm{SO}_{2} \times 97.5 \%{ }^{\mathrm{d}}$ & 4650.3 \\
\hline & & ind & 2937.5 & Ind $\mathrm{SO}_{2}$ x $97.5 \%$ & 7826.3 \\
\hline & & forest fire & 7.0 & - & 7.0 \\
\hline & & grass fire & 1.4 & - & 1.4 \\
\hline & & contvolc & 0.0 & - & 0.0 \\
\hline \multirow{8}{*}{$\begin{array}{l}\text { Acc. mode } \\
\text { sulfate }\end{array}$} & Surface & awb & 0.8 & - & 0.8 \\
\hline & & wst & 0.0 & - & 0.0 \\
\hline & & shp & 0.3 & - & 0.3 \\
\hline & Elevated & ene & 203.3 & pow $\mathrm{SO}_{2} \times 2.5 \%$ & 119.2 \\
\hline & & ind & 75.3 & ind $\mathrm{SO}_{2} \times 2.5 \%$ & 200.7 \\
\hline & & forest fire & 0.2 & - & 0.2 \\
\hline & & grass fire & 0.0 & - & 0.0 \\
\hline & & contvolc & 0.0 & - & 0.0 \\
\hline Ait. mode & Surface & dom & 43.4 & res $\mathrm{SO}_{2} \times 2.5 \%$ & 40.9 \\
\hline
\end{tabular}




\begin{tabular}{|c|c|c|c|c|c|}
\hline sulfate & & tra & 7.4 & $\operatorname{tra} \mathrm{SO}_{2} \times 2.5 \%$ & 2.6 \\
\hline & Elevated & contvolc & 0.0 & - & 0.0 \\
\hline Total sulfur & & & 13246.0 & & 14585.2 \\
\hline \multirow[t]{9}{*}{ BC } & Surface & ene & 20.5 & pow BC & 1.9 \\
\hline & & ind & 853.8 & Ind $\mathrm{BC}$ & 546.4 \\
\hline & & dom & 581.9 & res $\mathrm{BC}$ & 881.3 \\
\hline & & tra & 74.7 & $\operatorname{tra} B C$ & 286.1 \\
\hline & & awb & 43.9 & - & 43.9 \\
\hline & & wst & 6.4 & - & 6.4 \\
\hline & & $\operatorname{shp}$ & 0.3 & - & 0.3 \\
\hline & Elevated & fst & 9.8 & - & 9.8 \\
\hline & & grs & 3.6 & - & 3.6 \\
\hline Total BC & & & 1595.0 & & 1779.8 \\
\hline \multirow[t]{9}{*}{ РОМ } & Surface & ene & 93.6 & pow OC x 1.4 & 0.0 \\
\hline & & ind & 1567.8 & ind $\mathrm{OC} \times 1.4$ & 707.5 \\
\hline & & dom & 2314.5 & res OC x 1.4 & 3778.8 \\
\hline & & tra & 129.5 & $\operatorname{tra} \mathrm{OC} \times 1.4$ & 148.3 \\
\hline & & awb & 292.8 & - & 292.8 \\
\hline & & wst & 9.0 & - & 9.0 \\
\hline & & $\operatorname{shp}$ & 0.5 & - & 0.5 \\
\hline & Elevated & fst & 185.3 & - & 185.3 \\
\hline & & grs & 36.2 & - & 36.2 \\
\hline Total POM & & & 4629.0 & & 5158.4 \\
\hline \multirow[t]{7}{*}{ SOAG } & Surface & BIGALK & 179.8 & $($ pow + ind + tra + res & 376.2 \\
\hline & & & & ALK3, ALK4, & \\
\hline & & & & ALK5)*molecular & \\
\hline & & & & weight *mass & \\
\hline & & & & yield $* 1.5^{\mathrm{g}}$ & \\
\hline & & BIGENE & 33.6 & (pow + ind $+\operatorname{tran}+$ res & 91.4 \\
\hline & & & & OLE2) *molecular & \\
\hline
\end{tabular}




\section{weight *mass}

yield*1.5

TOLUENE

ISOPRENE

TERPENE
352.8

712.6

1289.6 (pow + ind + tra + res

ARO1, ARO2)

*molecular weight

*mass yield*1.5

Total SOAG

2568.3

1031.9

1289.6

3501.6
DMS
Surface
8.2
8.2

$\overline{{ }^{a}}$ The AR5 sector abbreviations are dom (domestic), tra (transportation), ind (industry), ene (energy), wst (waste treatment), awb (agricultural waste burning), shp (shipping), fst (forestfire), grs (grass fire)and contvolc (continuous volcano).

$10{ }^{\mathrm{b}}$ The MEIC sector abbreviations are res (residential), tra (transportation), ind (industry), and pow(power). c " "." means that the species in the sector is the same as AR5 emission.

${ }^{d}$ The elevated energy and industry emissions are emitted in mass fraction of $15.5 \%, 75.1 \%$, and $9.4 \%$ at approximately 30 , 130 , and 280 meters.

${ }^{\mathrm{e}}$ The masses of $\mathrm{SO}_{2}$, sulfate and DMS are in unit of Gg of Sulfur per year in China. The unit of BC mass is Gg of Carbon

15 per year and the units of POM and SOAG mass are Gg of POM per year, which is assumed to be 1.4 times OC (or Carbon) mass.

${ }^{\mathrm{f}}$ The units are the same as in the column of AR5 emission rates.

${ }^{g}$ Atmoic compositions for BIGALK, BIGENE, TOLUENE are $\mathrm{C}_{5} \mathrm{H}_{12}, \mathrm{C}_{4} \mathrm{H}_{8}$, and $\mathrm{C}_{6} \mathrm{H}_{5}\left(\mathrm{CH}_{3}\right)$, respectively. Mass yields of BIGALK, BIGENE, and TOLUENE are 5\%, 5\%, 15\%, respectively. 


\section{Observed surface concentration of aerosol in China}

Table S2. The observations of surface concentrations of chemical species in $\mathbf{P M}_{2.5}$ over eastern China in 2009 and 2010.

\begin{tabular}{|c|c|c|c|c|c|}
\hline Locations & Coordinates & Time & Chemical species & References & Location type \\
\hline Harbin & $\begin{array}{l}45.82^{\circ} \mathrm{N} \\
126.56^{\circ} \mathrm{E}\end{array}$ & Aug-Dec, 2010 & $\mathrm{SO}_{4}, \mathrm{BC}, \mathrm{OC}$ & Huang et al.[2014] & urban \\
\hline Chengde & $\begin{array}{l}40.95^{\circ} \mathrm{N} \\
117.96^{\circ} \mathrm{E}\end{array}$ & $\begin{array}{l}\text { April/July/Oct, } \\
\text { 2009;Jan, } 2010\end{array}$ & $\mathrm{SO}_{4}, \mathrm{BC}, \mathrm{OC}$ & Zhao et al. [2013] & urban \\
\hline Shangdianzi & $\begin{array}{l}38.04^{\circ} \mathrm{N} \\
114.51^{\circ} \mathrm{E}\end{array}$ & $\begin{array}{l}\text { April/July/Oct, } \\
\text { 2009;Jan, } 2010\end{array}$ & $\mathrm{SO}_{4}, \mathrm{BC}, \mathrm{OC}$ & Zhao et al. [2013] & rurual \\
\hline Beijing1 & $\begin{array}{l}39.93^{\circ} \mathrm{N} \\
116.30^{\circ} \mathrm{E}\end{array}$ & $\begin{array}{l}\text { April/July/Oct, } \\
\text { 2009;Jan, } 2010\end{array}$ & $\mathrm{SO}_{4}, \mathrm{BC}, \mathrm{OC}$ & Zhao et al. [2013] & urban \\
\hline Beijing2 & $\begin{array}{l}39.99^{\circ} \mathrm{N} \\
116.30^{\circ} \mathrm{E}\end{array}$ & $\begin{array}{l}\text { April/July/Oct, } \\
\text { 2009;Jan, } 2010\end{array}$ & $\mathrm{SO}_{4}, \mathrm{BC}, \mathrm{OC}$ & Zhang et al. [2013] & urban \\
\hline Tianjin & $\begin{array}{l}39.08^{\circ} \mathrm{N} \\
117.20^{\circ} \mathrm{E}\end{array}$ & $\begin{array}{l}\text { April/July/Oct, } \\
\text { 2009;Jan, } 2010\end{array}$ & $\mathrm{SO}_{4}, \mathrm{BC}, \mathrm{OC}$ & Zhao et al. [2013] & urban \\
\hline $\begin{array}{l}\text { Shijiazhuan } \\
\mathrm{g}\end{array}$ & $\begin{array}{l}38.04^{\circ} \mathrm{N} \\
114.51^{\circ} \mathrm{E}\end{array}$ & $\begin{array}{l}\text { Apri1/July/Oct, } \\
\text { 2009;Jan, } 2010\end{array}$ & $\mathrm{SO}_{4}, \mathrm{BC}, \mathrm{OC}$ & Zhao et al. [2013] & urban \\
\hline Zhengzhou & $\begin{array}{l}34.80^{\circ} \mathrm{N} \\
113.50^{\circ} \mathrm{E}\end{array}$ & $\begin{array}{l}\text { April/July/Oct, } \\
\text { 2009;Jan, } 2010\end{array}$ & $\mathrm{SO}_{4}, \mathrm{BC}, \mathrm{OC}$ & Geng et al. [2013] & urban \\
\hline \multirow[t]{2}{*}{ Shanghai } & $\begin{array}{l}31.18^{\circ} \mathrm{N} \\
121.42^{\circ} \mathrm{E}\end{array}$ & Jan, 2009 & $\mathrm{SO}_{4}, \mathrm{BC}, \mathrm{OC}$ & Feng et al. [2012] & urban \\
\hline & $\begin{array}{l}31.25^{\circ} \mathrm{N} \\
121.46^{\circ} \mathrm{E}\end{array}$ & $\begin{array}{l}\text { Oct, 2005; } \\
\text { Jan/Apr/July,2006 }\end{array}$ & $\mathrm{SOA}$ & Feng et al. [2009] & $\begin{array}{l}\text { urban, } \\
\text { suburban }\end{array}$ \\
\hline Wuhan & $\begin{array}{l}30.50^{\circ} \mathrm{N} \\
114.3^{\circ} \mathrm{E}\end{array}$ & $\begin{array}{l}\text { Aug 2012- July } \\
2013\end{array}$ & SOA & Zhang et al. [2015] & $\begin{array}{l}\text { urban, } \\
\text { suburban }\end{array}$ \\
\hline Chengdu ${ }^{*}$ & $\begin{array}{l}30.65^{\circ} \mathrm{N} \\
104.00^{\circ} \mathrm{E}\end{array}$ & Apr/May, 2009 & $\mathrm{SO}_{4}, \mathrm{BC}, \mathrm{OC}$ & Tao et al., [2013] & urban \\
\hline Xiamen & $\begin{array}{l}24.58^{\circ} \mathrm{N} \\
118.09^{\circ} \mathrm{E}\end{array}$ & $\begin{array}{l}\text { Jun,2009- } \\
\text { May,2010 }\end{array}$ & $\mathrm{SO}_{4}, \mathrm{BC}, \mathrm{OC}$ & Zhang et al. [2012] & urban \\
\hline \multirow[t]{2}{*}{ Guangzhou } & $\begin{array}{l}23.10^{\circ} \mathrm{N} \\
113.3^{\circ} \mathrm{E}\end{array}$ & $\begin{array}{l}\text { April/July/Oct, } \\
\text { 2009;Jan, } 2010\end{array}$ & $\mathrm{SO}_{4}, \mathrm{BC}, \mathrm{OC}$ & Tao et al. [2014] & urban \\
\hline & $\begin{array}{l}23.70^{\circ} \mathrm{N} \\
113.6^{\circ} \mathrm{E}\end{array}$ & $\begin{array}{l}\text { Mar,2012- } \\
\text { Mar,2013 }\end{array}$ & SOA & Lai et al.[2015] & rurual \\
\hline
\end{tabular}


* Tao et al. [2013] highlights the importance of the dust and biomass burning episodes to the chemical composition of PM2.5. We use their data on non-episodic days.

\section{Observed aerosol direct radiative effects in China}

Table S3. Aerosol direct radiative effects (ADREs) at TOA, surface (SFC), and within the atmosphere (ATM) in different regions and periods in China.

\begin{tabular}{|c|c|c|c|c|c|}
\hline Region & References & Period & $\begin{array}{l}\text { TOA } \\
\left(\mathrm{W} \mathrm{m}^{-2}\right)\end{array}$ & $\begin{array}{l}\text { SFC } \\
\left(\mathrm{W} \mathrm{m}^{-2}\right)\end{array}$ & $\begin{array}{l}\text { ATM } \\
\left(\mathrm{W} \mathrm{m}^{-2}\right)\end{array}$ \\
\hline CSHNET & Li et al. (2010) & Jan.-Dec. 2005 & & & \\
\hline Ansai $\left(36.85^{\circ} \mathrm{N}, 109.31^{\circ} \mathrm{E}\right)$ & Xin et al. (2007) & & -0.46 & -12.08 & 12.58 \\
\hline Beijing $\left(39.97^{\circ} \mathrm{N}, 116.37^{\circ} \mathrm{E}\right)$ & & & -3.30 & -30.60 & 27.30 \\
\hline Beijing Forest $\left(39.96^{\circ} \mathrm{N}, 115.43^{\circ} \mathrm{E}\right)$ & & & -0.91 & -7.59 & 6.66 \\
\hline Changbai Mt. $\left(42.40^{\circ} \mathrm{N}, 128.63^{\circ} \mathrm{E}\right)$ & & & & -6.67 & 6.82 \\
\hline Eerduosi $\left(39.48^{\circ} \mathrm{N}, 110.18^{\circ} \mathrm{E}\right)$ & & & & -6.02 & 6.02 \\
\hline Fengqiu $\left(35.00^{\circ} \mathrm{N}, 114.40^{\circ} \mathrm{E}\right)$ & & & -0.12 & -14.34 & 14.22 \\
\hline Fukang $\left(44.28^{\circ} \mathrm{N}, 87.92^{\circ} \mathrm{E}\right)$ & & & 2.03 & -5.80 & 7.80 \\
\hline Haibei $\left(37.45^{\circ} \mathrm{N}, 101.32^{\circ} \mathrm{E}\right)$ & & & & & 3.57 \\
\hline Hailun $\left(47.43^{\circ} \mathrm{N}, 126.63^{\circ} \mathrm{E}\right)$ & & & & -6.78 & 7.06 \\
\hline Jiaozhou Bay $\left(35.90^{\circ} \mathrm{N}, 120.18^{\circ} \mathrm{E}\right)$ & & & -2.81 & -24.12 & 21.31 \\
\hline Lanzhou $\left(36.07^{\circ} \mathrm{N}, 103.82^{\circ} \mathrm{E}\right)$ & & & & -22.29 & 21.94 \\
\hline Lhasa $\left(29.67^{\circ} \mathrm{N}, 91.33^{\circ} \mathrm{E}\right)$ & & & & -4.28 & 4.83 \\
\hline Sanjiang $\left(47.58^{\circ} \mathrm{N}, 133.52^{\circ} \mathrm{E}\right)$ & & & 0.93 & -6.92 & 7.85 \\
\hline Shanghai $\left(31.12^{\circ} \mathrm{N}, 121.75^{\circ} \mathrm{E}\right)$ & & & & -24.26 & 25.09 \\
\hline Shapotou $\left(37.45^{\circ} \mathrm{N}, 104.95^{\circ} \mathrm{E}\right)$ & & & & -7.45 & 7.42 \\
\hline Shenyang $\left(41.52^{\circ} \mathrm{N}, 123.63^{\circ} \mathrm{E}\right)$ & & & & -14.58 & 16.15 \\
\hline Taihu $\left(31.40^{\circ} \mathrm{N}, 120.22^{\circ} \mathrm{E}\right)$ & & & -2.64 & -15.79 & 13.15 \\
\hline Taoyuan $\left(28.92^{\circ} \mathrm{N}, 111.45^{\circ} \mathrm{E}\right)$ & & & 0.35 & & 19.95 \\
\hline Xianghe $\left(39.75^{\circ} \mathrm{N}, 116.96^{\circ} \mathrm{E}\right)$ & & & -1.28 & -28.78 & 27.50 \\
\hline Yanting $\left(31.27^{\circ} \mathrm{N}, 105.45^{\circ} \mathrm{E}\right)$ & & & 1.26 & -29.61 & 30.78 \\
\hline Xishuangbanna $\left(21.9^{\circ} \mathrm{N}, 101.27^{\circ} \mathrm{E}\right)$ & & & 2.40 & -18.17 & 20.55 \\
\hline \multicolumn{6}{|l|}{ Others } \\
\hline Xianghe $\left(39.75^{\circ} \mathrm{N}, 116.96^{\circ} \mathrm{E}\right)$ & Li et al. (2007) & Jan.-Dec. 2004-2005 & & -24 & \\
\hline Beijing $\left(39.98^{\circ} \mathrm{N}, 116.38^{\circ} \mathrm{E}\right)$ & Xia et al. (2007a) & Dec.-Feb. & -8.0 & -20.3 & \\
\hline
\end{tabular}




\begin{tabular}{|c|c|c|c|c|c|}
\hline & & Mar.-May & -13.9 & -46.1 & \\
\hline & & Jun.-Aug. & -13.5 & -45.6 & \\
\hline & & Sep.-Nov. & -10.7 & -30.0 & \\
\hline & & $2001-2005$ & & & \\
\hline Liaozhong $\left(41.50^{\circ} \mathrm{N}, 120.70^{\circ} \mathrm{E}\right)$ & Xia et al. (2007b) & Mar.-May 2005 & & -30 & \\
\hline Taihu $\left(31.70^{\circ} \mathrm{N}, 120.36^{\circ} \mathrm{E}\right)$ & Xia et al. (2007c) & Jan.-Dec. 2005-2006 & 0 & -38.4 & \\
\hline Nanjing $\left(32.05^{\circ} \mathrm{N}, 118.78^{\circ} \mathrm{E}\right)$ & Zhuang et al. (2014) & Jan.-Dec. 2011-2012 & -6.9 & -21.3 & \\
\hline $\operatorname{SACOL}\left(35.95^{\circ} \mathrm{N}, 104.10^{\circ} \mathrm{E}\right)$ & Liu et al. (2011) & May 2009 & -7.78 & -38.45 & 30.68 \\
\hline
\end{tabular}

\section{References}

Feng, J., Sun, P., Hu, X., Zhao, W., Wu, M., and Fu, J.: The chemical composition and sources of $\mathrm{PM}_{2.5}$ during the 2009 Chinese New Year's holiday in Shanghai, Atmos. Res., 118, 435-444, 2012.

Feng, Y., Chen, Y., Guo, H., Zhi, G., Xiong, S., Li, J., Sheng, G., and Fu, J.: Characteristics of organic and elemental carbon in $\mathrm{PM}_{2.5}$ samples in Shanghai, China, Atmos. Res., 92(4), 434-442, 2009.

35 Geng, N., Wang, J., Xu, Y., Zhang, W., Chen, C., and Zhang, R.: $\mathrm{PM}_{2.5}$ in an industrial district of Zhengzhou, China: chemical composition and source apportionment, Particuology, 11(1), 99-109, 2013.

Huang, L. and Wang, G.: Chemical characteristics and source apportionment of atmospheric particles during heating period in Harbin, China, J. Environ. Sci., 26(12), 2475-2483, 2014,.

Lai, S., Zhao, Y., Ding, A., Zhang, Y., Song, T., Zheng, J., Ho, K. F., Lee, S.-C., and Zhong, L.: Characterization of $\mathrm{PM}_{2.5}$ and the major chemical components during a 1-year campaign in rural Guangzhou, Southern China, Atmos. Res., 167, 208-215, 2016.

Li, Z., Xia, X., Cribb, M, Mi,W., Holben,B., Wang, P., Chen, H., Tsay S. C., Eck, T. F., Zhao, F., Dutton, E. G., and Dickerson, R. E.: Aerosol optical properties and their radiative effects in northern China, J. Geophys. Res., 112, D22S01, doi:10.1029/2006JD007382, 2007.

Li, Z., Lee, K. H., Wang, Y., Xin, J., Hao, W.-M.: First observation based estimates of cloud free aerosol radiative forcing across China, J. Geophys. Res.-Atmos., 115(D7), 2010.

Liu, Y., Huang, J., Shi, G., Takamura, T., Khatri, P., Bi, J., Shi, J., Wang, T., Wang, X., and Zhang, B.: Aerosol optical properties and radiative effect determined from skyradiometer over Loess Plateau of Northwest China, Atmos. Chem. Phys., 11, 11455-11463, 2011.

50 Tao, J., Zhang, L., Engling, G., Zhang, R., Yang, Y., Cao, J., Zhu, C., Wang, Y., and Luo, L.: Chemical composition of $\mathrm{PM}_{2.5}$ in an urban environment in Chengdu, China: importance of springtime dust storms and biomass burning[, Atmos. Res., 122, 270-283, 2013. 
Tao, J., Zhang, L., Ho, K., Zhang, R., Lin, Z., Zhang, Z., Lin, M., Cao, J., Liu, S., and Wang, G.: Impact of PM $\mathrm{P}_{2.5}$ chemical compositions on aerosol light scattering in Guangzhou-the largest megacity in South China, Atmos. Res, 135, 48-58, 2014,.

Xia, X., Chen, H., Goloub, P., Zhang, W., Chatenet, B., and Wang, P.: A complicaiton of aerosol optical properties and calculation of direct radiative forcing over an urban region in northern China, J. Geophy. Res., 112, d12203, doi:10.1029/2006JD008119, 2007a.

Xia, X., Chen, H., Li, Z., Wang, P., and Wang J.: Significant reduction of surface solar irradiance induced by aerosols in a suburban region in northeastern China, J. Geophys. Res., 112, doi:10.1029/2006JD007562, 2007b.

Xia, X., Li, Z., Holben, B., Wang, P., Eck, T., Chen, H., Cribb, M., and Zhao, Y.: Aerosol optical properties and radiative effects in the Yangtze Delta region of China, J. Geophys. Res., 112, D22S12, 2007c.

Zhang, F., Xu, L., Chen, J., Yu, Y., Niu, Z., and Yin, L.: Chemical compositions and extinction coefficients of $\mathrm{PM}_{2.5}$ in peri-urban of Xiamen, China, during June 2009-May 2010, Atmos. Res., 106, 150-158, 2012.

Zhang, R., Jing, J., Tao, J., Hsu, S.-C., Wang, G., Cao, J., Lee, C. S. L., Zhu, L., Chen, Z., Zhao, Y., and Shen, Z.: Chemical characterization and source apportionment of $\mathrm{PM}_{2.5}$ in Beijing: seasonal perspective, Atmos. Chem. Phys., 13(14), 7053-7074, 2013.

Zhao, P. S., Dong, F., He, D., Zhao, X. J., Zhang, X. L., Zhang, W. Z., Yao, Q., and Liu, H. Y.: Characteristics of concentrations and chemical compositions for $\mathrm{PM}_{2.5}$ in the region of Beijing, Tianjin, and Hebei, China, Atmos.

70 Chem. Phys., 13, 4631-4644, 2013.

Zhuang, B.L., Wang, T.J., Li, S., Liu, J., Talbot, R., Mao, H.T., Yang, X.Q., Fu, C.B., Yin, C.Q., Zhu, J. L., Che, H.Z., and Zhang, X.Y.: Optical properties and radiative forcing of urban aerosols in Nanjing, China, Atmos. Environ., 83, 43-52, 2014. 